ARTICLE

DOI: $10.1038 / s 41467-018-07614-y$

\title{
Frequency-tunable toughening in a polymer-metal- ceramic stack using an interfacial molecular nanolayer
}

\author{
Matthew Kwan ${ }^{1}$, Muriel Braccini (iD) ${ }^{1,2}$, Michael W. Lane ${ }^{3}$ \& Ganpati Ramanath ${ }^{1}$
}

Interfacial toughening in composite materials is reasonably well understood for static loading, but little is known for cyclic loading. Here, we demonstrate that introducing an interfacial molecular nanolayer at the metal-ceramic interface of a layered polymer-metal-ceramic stack triples the fracture energy for $\sim 75-300 \mathrm{~Hz}$ loading, yielding $40 \%$ higher values than the static-loading fracture energy. We show that this unexpected frequency-dependent toughening is underpinned by nanolayer-induced interface strengthening, which facilitates load transfer to, and plasticity in, the polymer layer. Above a threshold interfacial bond strength, the toughening magnitude and frequency range are primarily controlled by the frequencyand temperature-dependent rheological properties of the polymer. These results indicate the tunability of the toughening behavior through suitable choice of interfacial molecular layers and polymers. Our findings open up possibilities for realizing novel composites with inorganic-organic interfaces, e.g., arresting crack growth or stimulating controlled fracture triggered by loads with specific frequency characteristics.

\footnotetext{
${ }^{1}$ Materials Science and Engineering Department, Rensselaer Polytechnic Institute, Troy, NY 12180, USA. ${ }^{2}$ SIMaP, Grenoble INP, CNRS, Univ. Grenoble Alpes, F-38000 Grenoble, France. ${ }^{3}$ Chemistry Department, Emory and Henry College, Emory, VA 24327, USA. Correspondence and requests for materials should be addressed to G.R. (email: Ramanath@rpi.edu)
} 
$\mathrm{T}$ ailoring the chemistry of heterointerfaces is crucial to controlling the fracture toughness of a variety of composite materials, such as, those used in load-bearing structures ${ }^{1}$, nanoelectronics devices ${ }^{2}$, energy systems ${ }^{3}$, and biomedicine ${ }^{4}$. Interfacial fracture can occur at significantly lower stresses than the static-loading fracture stresses ${ }^{5,6}$ of the materials comprising the interface, and can be exacerbated by chemical attack (stress corrosion) and cyclic loading (fatigue), thereby adversely impacting reliability and performance. Although fatigue has been widely investigated and well understood in bulk materials $\mathrm{s}^{7,8}$, much less is known about fatigue-induced interfacial fracture, especially in coatings and thin films. Recent works have examined the effects of chemical treatment ${ }^{9,10}$, patterning ${ }^{11}$, micrometerthick adhesion layers ${ }^{4,10}$, crack-tip blunting ${ }^{12}$, and cyclic loading amplitude ${ }^{13}$ on interfacial fatigue, and described the results in terms of Paris law-based bulk-fatigue models ${ }^{14,15}$. But, loadingfrequency-dependence of interfacial fatigue, and related phenomena, remain largely unexplored. Our prior work has shown that introducing an interfacial molecular nanolayer (MNL) in model polymer-metal-ceramic structures can yield multifold increases in fracture energy under static loading ${ }^{16-19}$, enhance thermal $^{20}$ and electronic ${ }^{21-25}$ transport, inhibit diffusion ${ }^{26-28}$, and alter phase formation ${ }^{29}$. However, the effects of cyclic loading on the fracture behavior of such molecularly modified model systems are not known. Understanding frequency-dependent effects in molecularly modified structures, such as, accelerated damage, crack growth mitigation, and interfacial healing, is not only of fundamental importance but also should enable the design of smart composites ${ }^{30-32}$ comprised of soft-hard and/or organic-inorganic interfaces for emerging applications in electronics, energy, and biomedicine.

Here we report loading-frequency-dependent multifold fracture toughening upon inserting a strongly binding $\mathrm{MNL}$ at the metal-ceramic interface of a layered polymer-metal-ceramic stack. The interfacial MNL results in up to threefold higher fracture energy in the $\sim 75-300 \mathrm{~Hz}$ range than the invariant value at other frequencies. We demonstrate that this remarkable behavior is underpinned by MNL-induced interface strengthening that enables load transfer to, and plasticity in, the distal polymer layer. Furthermore, the magnitude and frequency range of fatigue toughening correlates with the rheological properties of the polymer, varied by altering the temperature relative to the polymer glass transition. Our findings suggest that fatigue fracture energy is tunable by appropriate choices of MNL(s) and polymer(s), opening up a completely new set of possibilities to tailor composite materials. For example, the interface can be tailored to shift from a crack growth mode to crack arrest mode, or controllably fracture in response to stimuli with specific loading-frequency characteristics.

\section{Results}

Experimental work. We prepared polymer-metal-MNL-ceramic structures sandwiched between two silica-capped $\mathrm{Si}(001)$ wafers for four-point-bend mechanical tests ${ }^{19}$ (Fig. 1a). We selfassembled a mercapto-propyl-tri-methoxysilane (MPTMS) MNL on a silica-capped Si wafer surface. We then sputterdeposited a 40-nm-thick $\mathrm{Cu}$ layer with a $7 \mathrm{mTorr}$ Ar plasma in a $5 \times 10^{-7}$ Torr base pressure CVC tool. Without breaking vacuum, we also deposited a 100 -nm-thick Ta layer to facilitate metalpolymer bonding ${ }^{17}$. We glued the metal-MNL-silica structures to a dummy Si wafer with a System Three Resins ${ }^{\circledR}$ T88 epoxy polymer to obtain beams comprised of layered Si-polymer-Ta$\mathrm{Cu}$-MPTMS-SiO ${ }_{2}-\mathrm{Si}$ structures for four-point-bend mechanical tests $^{19}$ (Fig. 1a). We also created and tested beams without the MNL (see Methods).

Four-point bend tests were carried out under fatigue and static loading at temperatures ranging from $15 \leq T \leq 50^{\circ} \mathrm{C}$ and at preset water partial pressures between $0.6 \leq p_{\mathrm{H}_{2} \mathrm{O}} \leq 2.8 \mathrm{kPa}$. A Physik Instrumente $\mathrm{P} 216-9 \mathrm{~S}$ piezo actuator was used to produce loadcycling. Following crack initiation of the $\mathrm{Cu}-\mathrm{SiO}_{2}$ interface $^{33}$ at a critical strain energy release rate $\left(\Gamma_{\text {Critical }}\right)$, we conducted displacement-controlled subcritical (i.e., $\quad \Gamma<\Gamma_{\text {Critical }}$ ) loadshedding tests ${ }^{18,34}$. Example stress-time curves from a loadshedding fatigue test, and a static-loading test, are schematically depicted in Fig. 1b. For the fatigue tests, we chose a displacementamplitude between 5 and $30 \mu \mathrm{m}$ to obtain an initial stress slightly higher than that corresponding to the fatigue fracture energy $\Gamma_{\text {Fatigue }}$ to be measured. We applied sinusoidal load oscillations in the $0.1 \leq v \leq 1000 \mathrm{~Hz}$ range, with a maximum-to-minimum load ratio $\sim 10$. We extracted the $\Gamma_{\text {Fatigue }}$ from strain energy release rate-crack velocity $\left(\Gamma-u_{\text {crack }}\right)$ plots by recognizing that $\Gamma=\Gamma_{\text {Fatigue }}$ at $u_{\text {crack }}=0$. We also determined the stress corrosion fracture energy $\Gamma_{\text {Static }}$ from static load tests ${ }^{19}$ to explicitly separate the effects of chemical attack and load-cycling. The fracture surfaces were examined by X-ray photoelectron spectroscopy (XPS) and polarized-light microscopy.

Loading-frequency-dependent toughening. Our results show that introducing a MPTMS nanolayer at the $\mathrm{Cu}-\mathrm{SiO}_{2}$ interface of our polymer-metal-silica stacks significantly influences the fatigue fracture behavior. Stacks without the MPTMS nanolayer exhibit a $\Gamma_{\text {Fatigue }} \sim \Gamma_{\text {Static }}=1.6 \mathrm{Jm}^{-2}$ at $25^{\circ} \mathrm{C}$ and $p_{\mathrm{H}_{2} \mathrm{O}}=0.6 \mathrm{kPa}$ (Fig. $1 \mathrm{c}$ ), irrespective of the loading frequency. Inserting an MPTMS

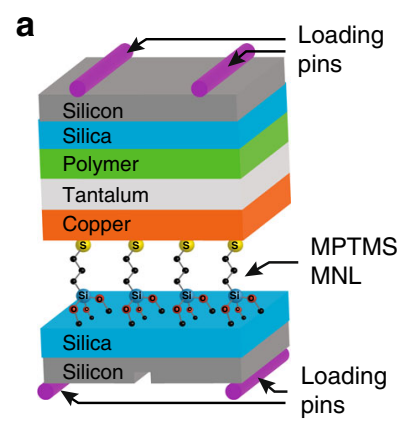

C

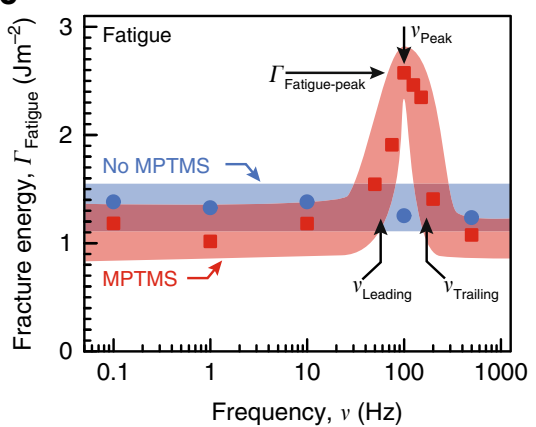

Fig. 1 Fracture energy of epoxy-Cu-MPTMS-SiO ${ }_{2}$ structures under static and fatigue loading. a Schematic depicting the four-point bending test and b the strain energy release rate characteristics for load-shedding fatigue and static loading. c Fatigue fracture energy of polymer-metal-ceramic structures with $\mathrm{Cu}-\mathrm{MPTMS}-\mathrm{SiO}_{2}$ interfaces (red squares) and $\mathrm{Cu}-\mathrm{SiO}_{2}$ interfaces (blue circles) determined at $p_{\mathrm{H}_{2} \mathrm{O}}=0.6 \mathrm{kPa}$, shown together with d the corresponding static stress fracture energies. Each data point represents at least three tests. The width of the bands, drawn through the data points to guide the eye, connote the experimental uncertainty measured as standard deviation 


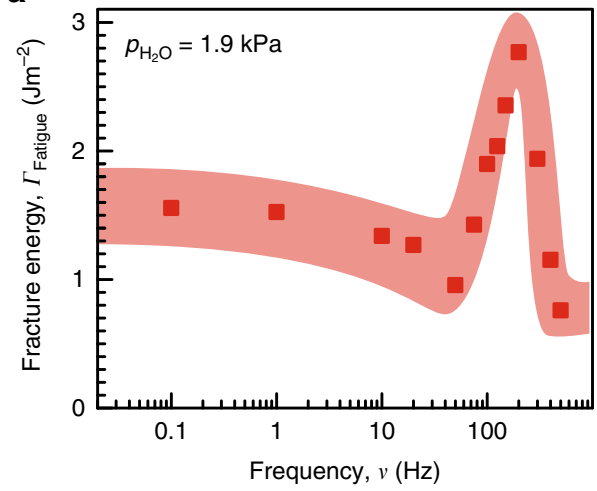

C

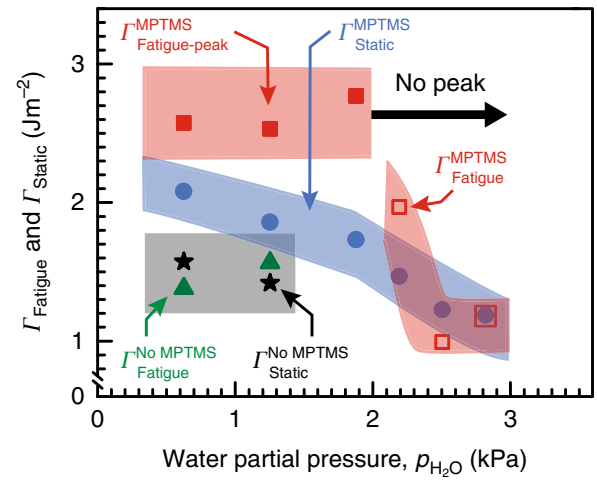

b

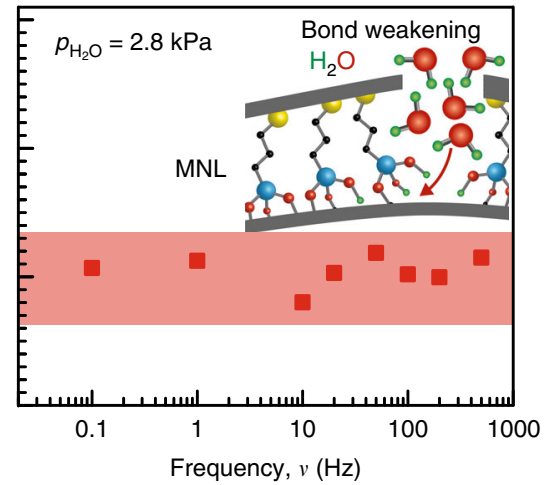

d

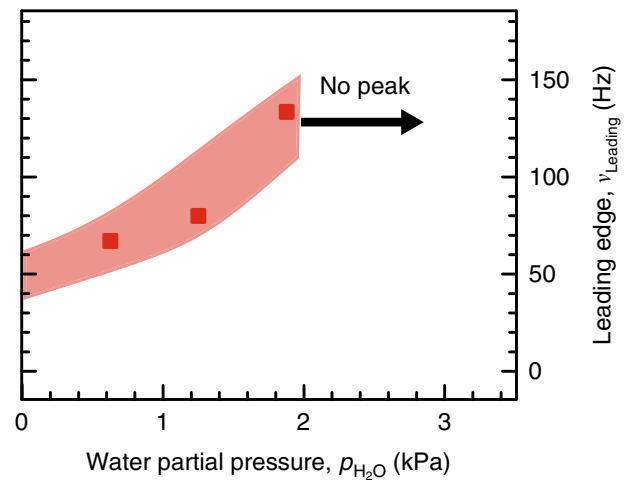

Fig. 2 Effect of moisture-dependent interfacial bond strength on fatigue toughening. Fatigue fracture energy $\Gamma_{\text {Fatigue }}$ of polymer-metal-ceramic structures with Cu-MPTMS-SiO ${ }_{2}$ interfaces shown for water partial pressures a $p_{\mathrm{H}_{2} \mathrm{O}}=1.9 \mathrm{kPa}$ and $\mathbf{b} p_{\mathrm{H}_{2} \mathrm{O}}=2.8 \mathrm{kPa}$. c $\Gamma_{\text {Fatigue }}$ peak value, i.e., $\Gamma_{\text {Fatigue-peak }}$ (red squares) and average $\Gamma_{\text {Static }}$ (blue circles) plotted versus $p_{\mathrm{H}_{2} \mathrm{O}}$ for stacks with Cu-MPTMS-SiO ${ }_{2}$ interfaces. Average $\Gamma_{\text {Fatigue }}$ (green triangles) and $\Gamma_{\text {Static }}$ (black stars) for stacks without MPTMS are also shown. There is no observable $\Gamma_{\text {Fatigue }}$ peak for $p_{\mathrm{H}_{2} \mathrm{O}}>2.2 \mathrm{kPa}$ (open red squares). d Leading edge frequency $\nu_{\text {Leading }}$ of the $\Gamma_{\text {Fatigue }}$ peak for Cu-MPTMS-SiO 2 interfaces. No $\Gamma_{\text {Fatigue }}$ peak is observed for $p_{\mathrm{H}_{2} \mathrm{O}} \geq 2.2 \mathrm{kPa}$. Each data point represents at least three tests. The width of the bands, drawn through the data points to guide the eye, connote the experimental uncertainty measured as standard deviation

nanolayer at the $\mathrm{Cu}-\mathrm{SiO}_{2}$ interface produces a $30 \%$ higher $\Gamma_{\text {Static }}$ $=2.1 \mathrm{Jm}^{-2}$, as expected ${ }^{35}$ (Fig. 1d). But, MPTMS functionalization decreases the fatigue fracture energy to $\Gamma_{\text {Fatigue }} \sim 1.1 \mathrm{Jm}^{-2}$ (i.e., $\left.\Gamma_{\text {Fatigue }}<\Gamma_{\text {Static }}\right)$ at all frequencies, except $v \sim 75-125 \mathrm{~Hz}$, where we observe $\Gamma_{\text {Fatigue }}>\Gamma_{\text {Static }}$ and a maximum of $\Gamma_{\text {Fatigue-peak }}$ $\sim 2.6 \mathrm{Jm}^{-2}$. In particular, $\Gamma_{\text {Fatigue }}$ increases above a threshold frequency $v_{\text {Leading, }}$, which we refer to as the leading edge (Fig. 1c). For $v>v_{\text {Leading, }}, \Gamma_{\text {Fatigue }}$ goes through a maximum at $v_{\max }$, and decreases at higher $v$. Fatigue toughening is not detectable for $v>$ $v_{\text {Trailing, }}$, where $v_{\text {Trailing }}$ corresponds to the trailing edge of the $\Gamma_{\text {Fatigue }}$ peak, i.e., where $\Gamma_{\text {Fatigue }}<\Gamma_{\text {Static }}$.

Our experiments revealing $\Gamma_{\text {Fatigue }}<\Gamma_{\text {Static }}$ at very low and very high load-cycling frequencies is not unexpected because subcritical cyclic loading is known to hasten interfacial fracture ${ }^{11}$. However, the observed loading-frequency-dependent toughening at intermediate frequencies (i.e., $v_{\text {Leading }} \leq v \leq v_{\text {Trailing }}$ ) indicated by the $\Gamma_{\text {Fatigue }}$ peak in the MPTMS-modified structures, is unusual. This result indicates that molecular functionalization of the weakest interface can actually increase the fracture energy at certain loading frequencies, to values higher than the staticloading fracture energy.

In order to understand the MPTMS-induced fatigue toughening, we measured the fracture energy as a function of the watersensitive siloxane bond strength ${ }^{36}$ at the $\mathrm{Cu}-\mathrm{MPTMS}-\mathrm{SiO}_{2}$ interface by adjusting the water partial pressure $p_{\mathrm{H}_{2} \mathrm{O}}$. For low

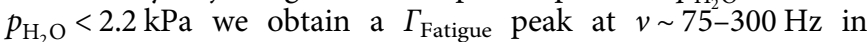
structures with $\mathrm{Cu}-\mathrm{MPTMS}-\mathrm{SiO}_{2}$ interfaces (Fig. 2a). At higher $p_{\mathrm{H}_{2} \mathrm{O}} \geq 2.2 \mathrm{kPa} \Gamma_{\text {Fatigue }}$ is essentially invariant in the $0.1 \leq v \leq 500$
$\mathrm{Hz}$ range, with no observable fatigue toughening (Fig. 2b), reflecting a behavior similar to that in structures without MPTMS. Since moisture weakens siloxane bonds, our results indicate a minimum interface bonding strength, attainable below a threshold $p_{\mathrm{H}_{2} \mathrm{O}}$, is a prerequisite for fatigue toughening. This is reminiscent of static toughening at $\mathrm{Cu}-\mathrm{MPTMS}-\mathrm{SiO}_{2}$ interfaces below a threshold $p_{\mathrm{H}_{2} \mathrm{O}}$ that provides adequate siloxane bonding strength to enable metal plasticity ${ }^{18,19}$.

Unlike a monotonic increase in the magnitude of static toughening with desiccation, fatigue toughening increases with desiccation in the $1.9 \mathrm{kPa} \leq p_{\mathrm{H}_{2} \mathrm{O}} \leq 2.5 \mathrm{kPa}$ range, but saturates at $\Gamma_{\text {Fatigue }}=\Gamma_{\text {Fatigue-peak }}$ for $p_{\mathrm{H}_{2} \mathrm{O}}<1.9 \mathrm{kPa}$ and is insensitive to further desiccation (Fig. 2c). We note that $\Gamma_{\text {Fatigue }}>\Gamma_{\text {Static }}$ at $p_{\mathrm{H}_{2} \mathrm{O}}=2.2 \mathrm{kPa}$, despite the absence of a $\Gamma_{\text {Fatigue peak. These }}$ observations suggest that the $\Gamma_{\text {Fatigue-peak }}$ magnitude is limited by a mechanism other than desiccation-induced interfacial siloxane bond strengthening. Fatigue toughening occurs only below a threshold $p_{\mathrm{H}_{2} \mathrm{O}}$ and only for $v \geq v_{\text {Leading, indicating that } v_{\text {Leading }}}$ corresponds to the threshold $p_{\mathrm{H}_{2} \mathrm{O}}$ at which the minimum required siloxane bond strength is attained at the crack tip. Toughening is precluded for $v<v_{\text {Leading }}$ because of facile waterinduced siloxane bond-breaking due to a higher $p_{\mathrm{H}_{2} \mathrm{O}}$ at the crack tip than the threshold $p_{\mathrm{H}_{2} \mathrm{O}}$. Conversely, $v>v_{\text {Leading }}$ corresponds to a lower $p_{\mathrm{H}_{2} \mathrm{O}}$ at the crack tip than the threshold $p_{\mathrm{H}_{2} \mathrm{O}}$. Thus, increasing frequency for $v>v_{\text {Leading }}$ (or equivalently, downshifting $v_{\text {Leading }}$ ) is tantamount to crack-tip desiccation. This is indeed corroborated by our results showing desiccation-induced downshifting of $v_{\text {Leading }}$ below $p_{\mathrm{H}_{2} \mathrm{O}} \leq 1.9 \mathrm{kPa}$ (Fig. 2d). 
a

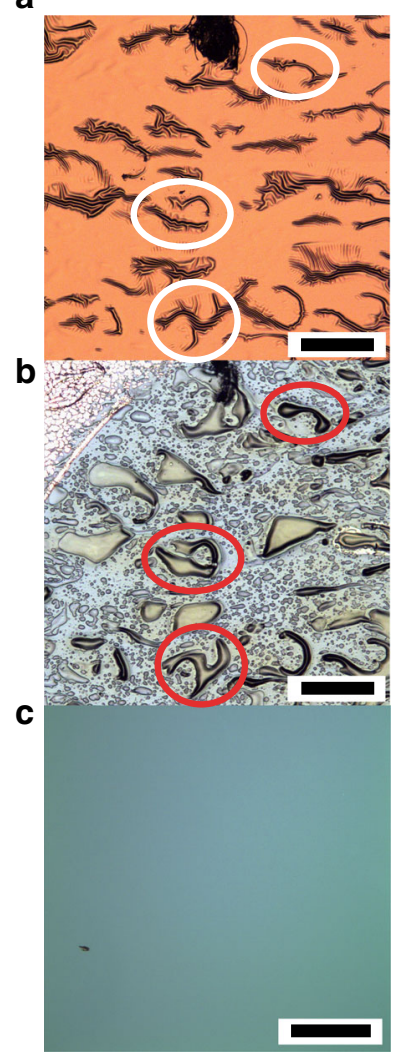

d
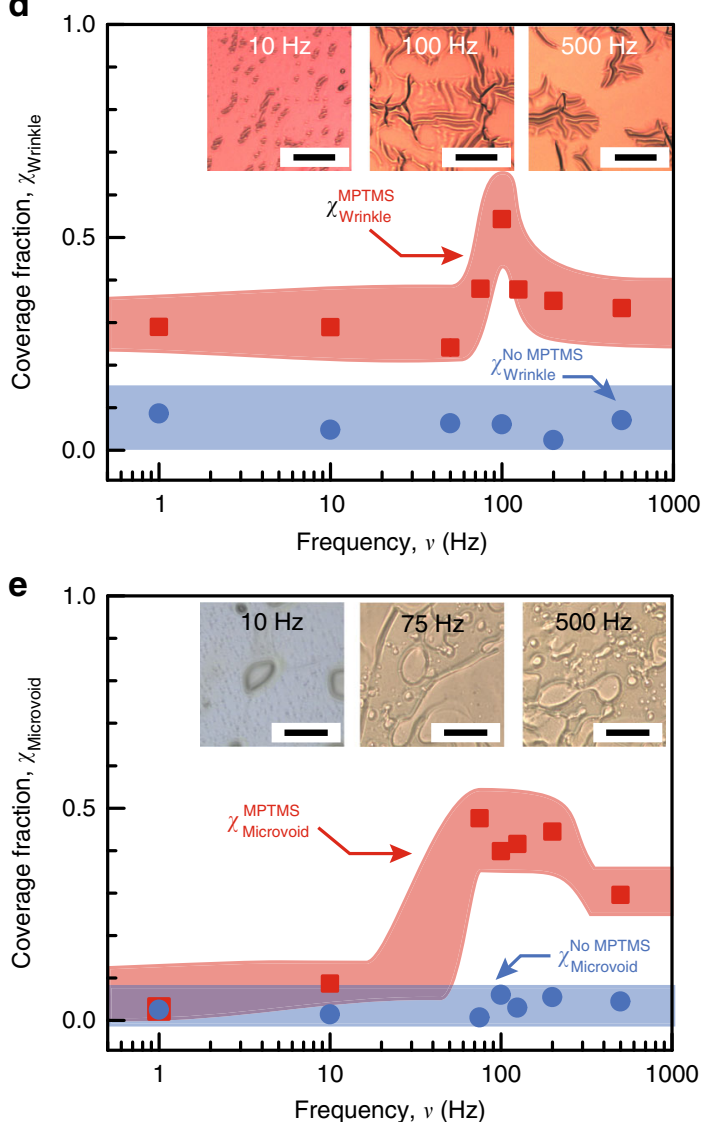

Fig. 3 Fracture surface analyses of epoxy-Cu-MPTMS- $\mathrm{SiO}_{2}$ structures under fatigue loading. a Representative optical micrographs from an as-obtained $\mathrm{Cu}$ fracture surface (scale bar $=300 \mu \mathrm{m}$ ), $\mathbf{b}$ the same with the metal film etched off to expose the polymer (scale bar $=300 \mu \mathrm{m}$ ), and $\mathbf{c}$ the $\mathrm{SiO}_{2}$ fracture surface (scale bar $=100 \mu \mathrm{m}$ ). The sizes and shapes of the metal wrinkles and the polymer microvoids are correlated (circles regions). Average $\mathbf{d}$ metal wrinkle coverage $\chi$ Wrinkle $\left(\right.$ scale bar $=100 \mu \mathrm{m}$ ) and $\mathbf{e}$ microvoid coverage $\chi_{\text {Microvoid }}$ (scale bar $=200 \mu \mathrm{m}$ ) from fracture surfaces of structures with (red squares), and without (blue circles) MPTMS. Representative optical micrographs inset in $\mathbf{d}$ and $\mathbf{e}$ capture metal wrinkling and polymer microvoiding at select frequencies. The data in this figure were from experiments at $p_{\mathrm{H}_{2} \mathrm{O}}=1.3 \mathrm{kPa}$. The width of the bands drawn through the data points connote the experimental uncertainties measured as standard deviation

The correlations between increasing $v$ and desiccation suggest that the water molecules are increasingly hindered from reaching the crack tip for $v>v_{\text {Leading, }}$ similar to that reported for high loading rates ${ }^{37-39}$. The build-up of elastic energy in the unbroken interfacial bonds increases the interfacial work of adhesion $\gamma_{\mathrm{a}}$ and becomes available for activating plastic energy dissipation $\gamma_{\mathrm{p}}$ in the adjacent layers. Thus, siloxane bond strength is the limiting determinant of the toughening magnitude with increasing frequency in the $v_{\text {Leading }} \leq v \leq v_{\text {peak }}$ range and desiccation in the $1.9 \mathrm{kPa} \leq p_{\mathrm{H}_{2} \mathrm{O}} \leq 2.8 \mathrm{kPa}$ range. Decreasing $\Gamma_{\text {Fatigue }}$ for $v>v_{\text {peak }}$ and the invariance of $\Gamma_{\text {Fatigue-peak }}$ magnitude for $p_{\mathrm{H}_{2} \mathrm{O}}<1.9 \mathrm{kPa}$ are contrary to desiccation-induced bond strengthening, confirming that $\Gamma_{\text {Fatigue }}$ at high $v$ and low $p_{\mathrm{H}_{2} \mathrm{O}}$ is not limited by the interfacial strength.

Interfacial fracture and plastic energy dissipation. Fracture surface analyses suggests that MPTMS-functionalization leads to fatigue toughening by facilitating plasticity in the metal-polymer bilayer. Core-level XPS spectra from fracture surfaces confirm that fatigue fracture occurs via siloxane bond-breaking at the MPTMS-silica interface (Supplementary Fig. 1), as reported for static loading ${ }^{17,18}$. Polarized-light micrographs of $\mathrm{Cu}$ fracture surfaces exhibit 5 - to 20 - $\mu$ m-scale wrinkles (Fig. 3a). Etching off the metal film reveals microvoids in the polymer that are similar in shape and size of the metal wrinkles at the same location (Fig. 3b), suggesting that polymer voiding and metal wrinkling are correlated. In contrast, the silica fracture surfaces were featureless (Fig. 3c), consistent with our XPS analysis. Load-cycling results increases the average microvoid area $\zeta_{\text {Microvoid }}$ multifold, e.g., from 175 to $980 \mu \mathrm{m}^{2}$ (Supplementary Fig. 2). Radially oriented polarization fringes around the microvoids (Supplementary Fig. 3) indicate microvoid growth by shear banding involving the back-and-forth motion of polymer chains ${ }^{9}$. Fracture surfaces obtained by static loading show neither metal wrinkling nor polymer microvoid growth, confirming that these features arise from load-cycling-induced plasticity.

Both metal wrinkling and polymer microvoid coverages ( $\chi_{\text {Wrinkle }}$ and $\left.\chi_{\text {Microvoid }}\right)$ are dependent on the loading frequency.

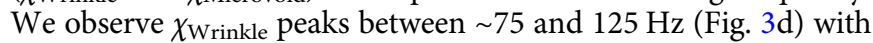
a $60 \%$ higher coverage than other frequencies, while $\chi_{\text {Microvoid }}$ increases eightfold and peaks over a wider frequency range of $\sim 75-200 \mathrm{~Hz}$, and saturates at a slightly lower value above $\sim 200$ $\mathrm{Hz}$ (Fig. 3e). The frequency regimes of the coverage peaks correlate well with the $75-300 \mathrm{~Hz}$ regime of the $\Gamma_{\text {Fatigue }}$ peak. In contrast, fatigue fracture surfaces of stacks without MPTMS exhibit ninefold lower $\chi_{\text {Microvoid }}$ and fourfold lower $\chi_{\text {Wrinkle }}$, both of which are invariant with frequency. These results confirm that 


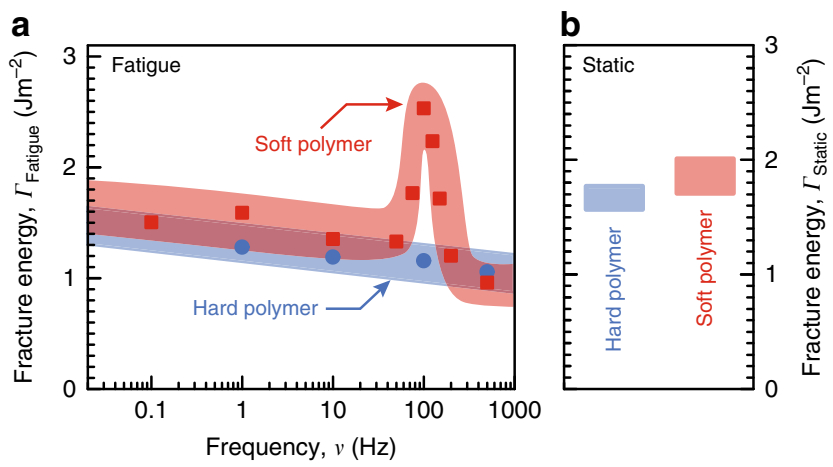

Fig. 4 Effect of polymer hardness on fatigue toughening. a Fatigue fracture energy $\Gamma_{\text {Fatigue }}$ and $\mathbf{b} \Gamma_{\text {Static }}$ of polymer-metal-MPTMS-SiO ${ }_{2}$ stacks with a soft System Three Resins ${ }^{\circledast}$ T88 polymer (red squares), and a harder EPOTEK 375 polymer (blue circles) at $p_{\mathrm{H}_{2} \mathrm{O}}=1.3 \mathrm{kPa}$. Each data point represents at least three tests. The width of the bands, drawn through the data points to guide the eye, connote the experimental uncertainties measured as standard deviation

MPTMS-induced interfacial strengthening is key to activating loading-frequency-dependent plasticity in the polymer-metal bilayer.

The greater overlap in the frequency regime of the $\chi_{\text {Microvoid }}$ peak and the $\Gamma_{\text {Fatigue }}$ peak suggests a greater contribution of polymer plasticity to the observed fatigue toughening. $\mathrm{Cu}$ film strain, estimated from $\chi_{\text {Wrinkle }}$ and wrinkle amplitude ${ }^{40}$ measurements (Supplementary Fig. 3), indicates a metal plastic energy $U_{\mathrm{m}} \sim 0.02 \pm 0.01 \mathrm{Jm}^{-2}$, which accounts for $<1 \%$ of the fracture energy at $\Gamma_{\text {Fatigue-peak }} \sim 2.5 \mathrm{Jm}^{-2}$ (Supplementary Methods). Such low metal plasticity is attributable to the high-yield stress $\mathrm{Cu}$ film that results in a highly confined plastic zone near the crack tip vicinity. This view is consistent with our estimates ${ }^{19}$ of $<\sim 2 \%$ plastic strain energy and a $<1 \mathrm{~nm}$ plastic zone for a 40 -nm-thick $\mathrm{Cu}$ film used in our experiments here. Thus, fatigue toughening observed in our experiments is underpinned primarily by polymer plasticity, while the metal film essentially serves as an elastic stress-transfer layer. We note that this mechanism is unlike MNL-induced metal-ceramic interface toughening due to metal plasticity under static loading ${ }^{19}$.

We propose that microvoid growth in the polymer leads to metal-polymer interface delamination at the voids. The consequent release of constraint leads to metal film wrinkling due to compressive stresses ${ }^{41}$. This hypothesis is supported by the disappearance of fatigue toughening and metal wrinkling when polymer plasticity is suppressed by replacing the T88 epoxy with the harder EPO-TEK 375 epoxy in our structures (Fig. 4 and Supplementary Fig. 3). The $\Gamma_{\text {Fatigue }}$ peak frequency range is also consistent with the toughening of T88 epoxy composites ${ }^{42-45}$ at strain rates of $\sim 0.02-0.1 \mathrm{~s}^{-1}$ (Supplementary Methods). If voiding were to initiate at the metal-polymer interface, we would expect significant metal wrinkling under static loading, and a fracture path change from the MNL-SiO ${ }_{2}$ interface, neither of which we observe. These results indicate that MPTMS-induced polymer plasticity is the primary fatigue toughening mechanism.

Tuning the toughening magnitude and frequency range. In order to understand fatigue toughening magnitude and frequency characteristics in terms of polymer rheology, we examined the $\Gamma_{\text {Fatigue }}$ peak at different temperatures below the polymer glass transition temperature $T_{\mathrm{g}}$. Since $T_{\mathrm{g}}$ for our T88 epoxy is frequency-dependent (e.g., $52 \leq T_{\mathrm{g}} \leq 75^{\circ} \mathrm{C}$ between $0.01 \leq v \leq$ $1000 \mathrm{~Hz}$ (Supplementary Fig. 4), we analyzed temperature- dependent toughening in terms of $\Delta T=T_{\mathrm{g}}-T$ for data acquired at a fixed $p_{\mathrm{H}_{2} \mathrm{O}}=1.3 \mathrm{kPa}$. Separate measurements of polymer film stress on $\mathrm{SiO}_{2}$ indicate that water-induced polymer swelling is insignificant, e.g., $<\sim 3 \%$ of the compliance change seen during crack growth (Supplementary Fig. 5).

We find that lower $\Delta T$ (i.e., higher $T$, closer to $T_{\mathrm{g}}$ ) correlates with a higher $\Gamma_{\text {Fatigue-peak }}$, and a larger peak width $\left(\Delta v=v_{\text {Trailing }}\right.$ $\left.-v_{\text {Leading }}\right)$, indicating that both the fatigue toughening magnitude and frequency range are sensitive to polymer plasticity (Fig. 5a). For instance, a $20^{\circ} \mathrm{C}$ increase in temperature from 16 to $36^{\circ} \mathrm{C}$ (i.e., decreasing $\Delta T$ from 59 to $39^{\circ} \mathrm{C}$ ) doubles the $\Gamma_{\text {Fatigue }}$ peak magnitude to $\Gamma_{\text {Fatigue-peak }}=2.9 \mathrm{Jm}^{-2}$ (Fig. $5 \mathrm{~b}$ ), which is $40 \%$ higher than the highest static-loading fracture energy $\Gamma_{\text {Static }}$ measured at $p_{\mathrm{H}_{2} \mathrm{O}}=0.6 \mathrm{kPa}$ (Fig. 2c). This $\Gamma_{\text {Fatigue-peak }}$ doubling correlates with the doubling of the loss-to-storage moduli ratio $\tan \delta$ of the epoxy for the same temperature increase (Supplementary Fig. 4), suggesting that polymer rheology determines the $\Gamma_{\text {Fatigue-peak }}$ magnitude. Since $\tan \delta$ increases with temperature for $T<T_{\mathrm{g}}$, but decreases with frequency (Supplementary Fig. $4 \mathrm{~b}-\mathrm{c}$ ), polymer plasticity is facilitated at higher temperatures but deterred at higher frequencies. Increasing $\Gamma_{\text {Fatigue-peak }}$ magnitude with temperature and decreasing $\Gamma_{\text {Fatigue }}$ for $v>v_{\text {Peak }}$ mirror the tan $\delta$ behavior, confirming that polymer plasticity is the predominant contributor to the observed fatigue toughening. Thus, decreasing polymer plasticity at $v>v_{\text {Peak }}$ counteracts increases in interfacial strength at $v>v_{\text {Leading, }}$, leading to a fatigue toughening maximum.

Decreasing $\Delta T$ (i.e., increasing $T$, closer to $T_{\mathrm{g}}$ ) also extends fatigue toughening to higher frequencies. Both $v_{\text {Leading }}$ and $v_{\text {Trailing }}$ shift to higher frequencies, but the $v_{\text {Trailing }}$ shift is $\sim 120 \%$ greater (Fig. $5 \mathrm{c}$ ). The temperature-induced $v_{\text {Trailing }}$ shifts can be understood by recognizing that plasticity is arrested above $v_{\text {Trailing }}$ due to the diminished responsiveness of polymer chains to highfrequency load-cycling, which is consistent with decreasing $\tan \delta$ with increasing frequency. Higher chain mobility at temperatures closer to $T_{\mathrm{g}}$ (i.e., high $T$ and low $\Delta T$ ) enables greater polymer plasticity, which is manifest as a higher $\Gamma_{\text {Fatigue-peak }}$ magnitude and shifting of the plasticity arrest point $v_{\text {Trailing }}$ to higher frequencies as $\Delta T$ decreases. Since $v_{\text {Leading }}$ corresponds to a threshold strength of the water-sensitive siloxane bonds, and $p_{\mathrm{H}_{2} \mathrm{O}}$ is held constant here, the up-shifts in $v_{\text {Leading }}$ with decreasing $\Delta T$ indicates that increasing the temperature enhances the transport of water molecules to the crack tip.

\section{Discussion}

Based upon our results, the salient mechanistic aspects of the frequency-dependent toughening observed in polymer-metalceramic structures with an MNL-functionalized metal-ceramic interface can be understood as follows. Interfacial fracture energy has two main contributors: the metal-ceramic interface work of adhesion $\gamma_{\mathrm{a}}$, and plastic energy dissipation $\gamma_{\mathrm{p}}$ in the adjacent layers. Introducing the MPTMS MNL increases $\gamma_{\mathrm{a}}$ through siloxane bond formation at the $\mathrm{MNL}-\mathrm{SiO}_{2}$ interface. Water attack of siloxane bonds lowers $\gamma_{\mathrm{a}}$. However, increasing load-cycling frequency curtails water transport to the crack tip, leading to an effective increase in $\gamma_{\mathrm{a}}$. Thus, increasing frequency is tantamount to desiccation, which strengthens siloxane bonds. The consequent build-up in interfacial elastic energy becomes available for activating plasticity (i.e., $\gamma_{p} \neq 0$ ) in the adjacent layers. The minimum $\gamma_{\mathrm{a}}$ necessary for elastic energy build-up and plastic energy dissipation for detectable fatigue toughening is achieved at $v_{\text {Leading, }}$ and increases for $v>v_{\text {Leading. }}$.

In our experiments, plasticity $\gamma_{\mathrm{p}}$ occurs mainly in the polymer by microvoid growth via shear banding. The high-yield stress metal film serves as an elastic load transfer layer, and wrinkles 


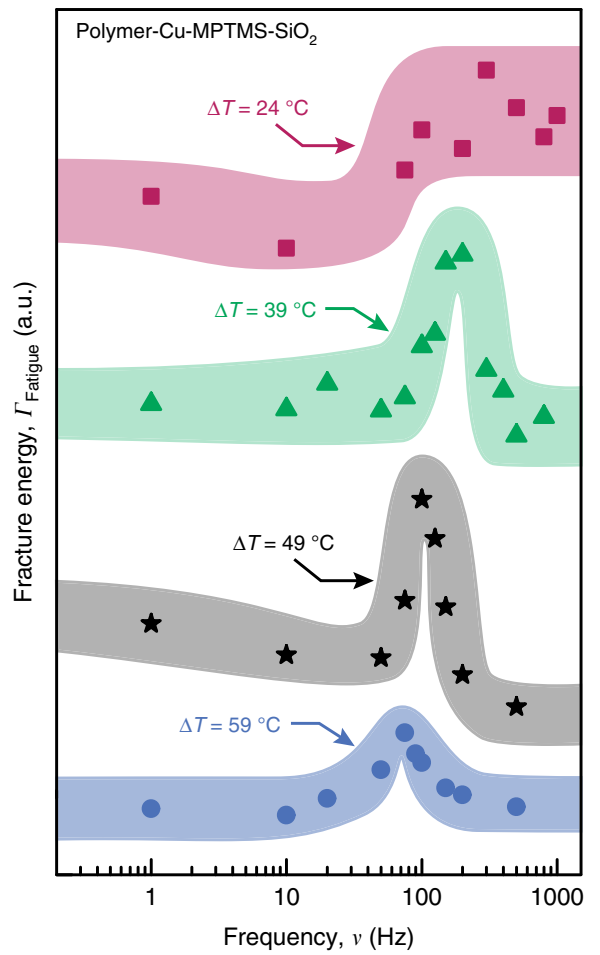

b
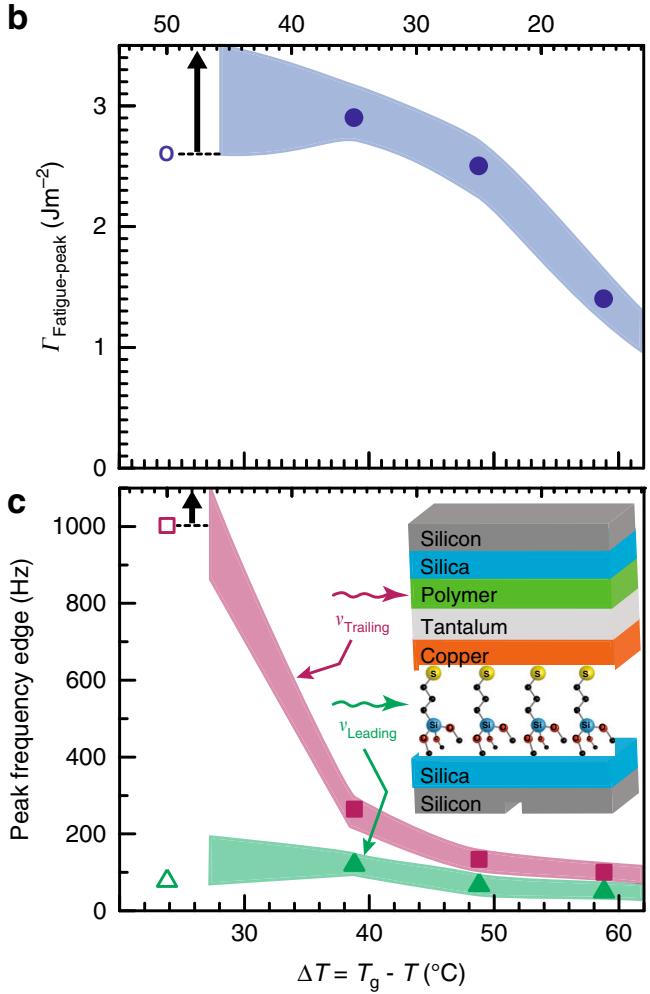

Fig. 5 Temperature-dependence of the fatigue toughening magnitude and frequency characteristics. a Fatigue fracture energy $\Gamma_{\text {Fatigue }}$ of polymer-metalMPTMS-silica interfaces for $24^{\circ} \mathrm{C} \leq \Delta T \leq 59^{\circ} \mathrm{C}$, at $p_{\mathrm{H}_{2} \mathrm{O}}=1.3 \mathrm{kPa}$. b The $\Gamma_{\text {Fatigue }}$ trailing edge frequency $\nu_{\text {Trailing }}$ (magenta squares), leading edge frequency $\nu_{\text {Leading }}$ (green triangles), and $\mathbf{c} \Gamma_{\text {Fatigue }}$ peak height $\Gamma_{\text {Fatigue-peak }}$ (blue circles), plotted as a function of $\Delta T$. The $\Gamma_{\text {Fatigue-peak }}$ and $\nu_{\text {Trailing }}$ values shown for $\Delta T=24{ }^{\circ} \mathrm{C}$ represent the lower bounds (open symbols) because the $\nu_{\text {Trailing }}$ edge at this temperature extends beyond our instrument frequency range. Each data point in $\mathbf{b}$ and $\mathbf{c}$ is extracted from the corresponding curve in $\mathbf{a}$. The width of the bands, drawn through the data points to guide the eye, connote the experimental uncertainties measured as standard deviation

due to compressive stresses generated through release of constraint when microvoids reach the polymer-metal interface. Polymer plasticity itself, however, decreases with increasing frequency due to the increasing inability of polymer chain motion to keep up with load-cycling. Increasing $\gamma_{\mathrm{a}}$ and decreasing $\gamma_{\mathrm{p}}$ with frequency results in a fatigue toughening maximum, i.e., a $\Gamma_{\text {Fatigue }}$ peak, at intermediate frequencies. The increase in $\Gamma_{\text {Fatigue }}$ with frequency for $v_{\text {leading }} \leq v \leq v_{\text {Peak }}$ indicates that fatigue toughening is limited by $\gamma_{\mathrm{a}}$. At $v \geq v_{\text {Peak }}$, the decreasing contribution of $\gamma_{\mathrm{p}}$ due to inhibited chain motion begins to offset the frequency-induced increases in the elastic energy due to interfacial bond strengthening. Decreasing fatigue toughening for $v>v_{\text {peak }}$ and saturation for $p_{\mathrm{H}_{2} \mathrm{O}}<1.9 \mathrm{kPa}$ are contrary to interfacial siloxane bond strengthening expected at high $v$ and low $p_{\mathrm{H}_{2} \mathrm{O}}$, confirming that fatigue toughening is limited by polymer plasticity at high frequencies.

Although bond strengthening $\gamma_{\mathrm{a}}$ through the use of the MNL is a necessary condition for fatigue toughening, polymer plasticity $\gamma_{\mathrm{p}}$ is the predominant contributor. This is clearly seen from the suppression of fatigue toughening in MNL-modified structures with a hard epoxy. Furthermore, the maximum toughening magnitude $\Gamma_{\text {Fatigue-peak }}$ and high-frequency limit of toughening $v_{\text {Trailing, }}$ are determined by the rheological properties of the polymer. For example, both $\Gamma_{\text {Fatigue-peak }}$ and $v_{\text {Trailing }}$ increase with temperature (as $T$ approaches $T_{\mathrm{g}}$ ) due to facile chain motion, which is counteracted to some extent by the frequency-induced increase in $T_{\mathrm{g}}$ at higher frequencies. Therefore, choosing a polymer with the appropriate rheological properties is crucial for tailoring the toughening magnitude as well as the high-frequency limit.

Based upon the above, the overall mechanism of fatigue toughening of the epoxy-Cu-MPTMS-SiO 2 polymer-metal-MNLceramic stack enabled by the MPTMS MNL can be understood in terms of Fig. 6. At low loading frequencies, water-induced siloxane bond-breaking at the MNL-ceramic interface limits the interface fracture energy. At intermediate loading frequencies, the increased interface strength caused by the diminishing effect of water attack at the $\mathrm{MNL}-\mathrm{SiO}_{2}$ interface facilitates polymer plasticity resulting in fracture energies exceeding the static-loading fracture energy. Arrested polymer plasticity due to curtailed chain mobility at very high loading frequencies leads to the disappearance of polymer plasticity, and hence, fatigue toughening. Although the metal serves as an elastic layer in our experiments, using metals of different yield stresses, moduli, and/or thicknesses, may alter the contributions of metal and polymer plasticity, which could amplify, suppress, and/or modify the fatigue toughening frequency range.

Our findings are relevant to the design, monitoring, and controlling the stability of smart composites with tunable frequencydependent toughening and/or weakening behaviors. For example, low-frequency fatigue toughening commencement $v_{\text {Leading }}$ in polymer-metal-MNL-ceramic structures can be controlled by suitable choice of MNLs with termini that enable strong interfacial bonding that supports sufficient interfacial elastic energy build-up for activating plasticity in the adjacent layers. Choosing polymers with appropriate viscoelastic properties should allow 


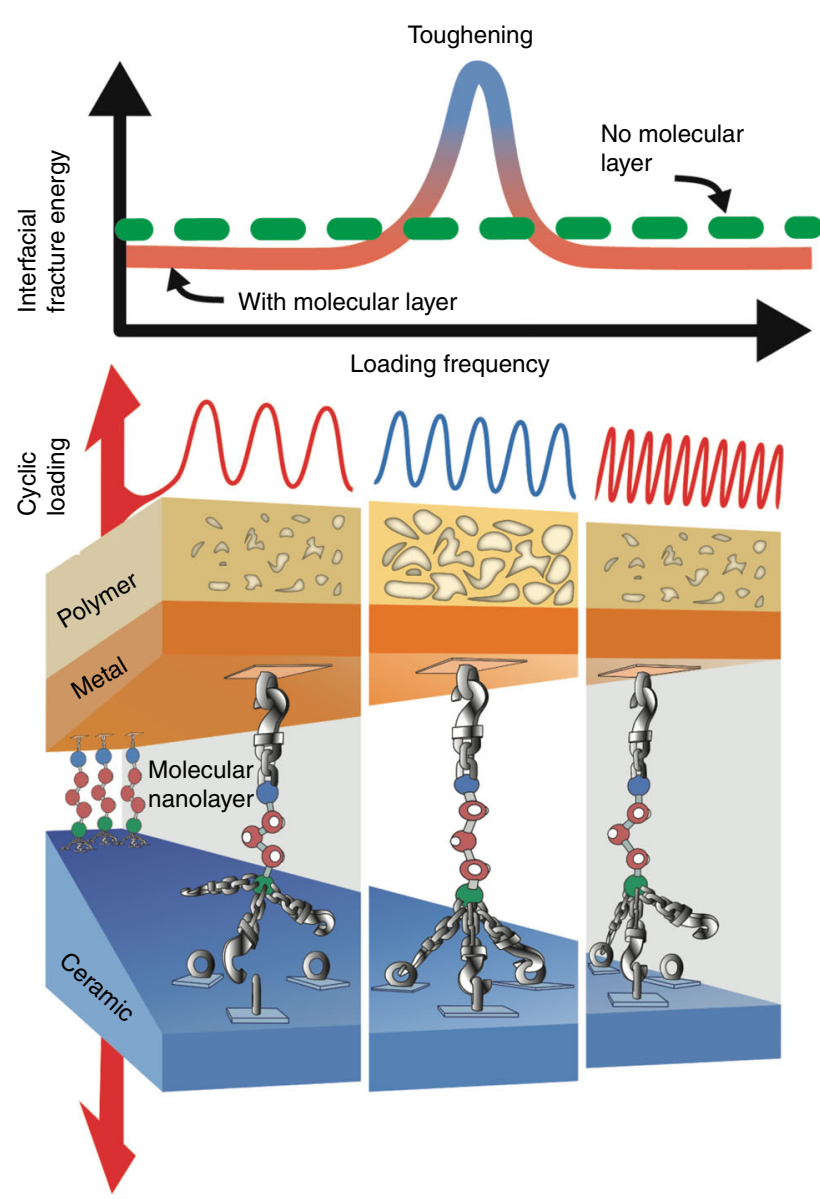

Fig. 6 Frequency-dependent toughening enabled by interfacial strengthening and polymer rheology. Schematic sketch of loadingfrequency-dependent interfacial fracture energy increase in an epoxy-CuMPTMS-SiO ${ }_{2}$ stack caused by plasticity in the polymer layer through voiding activated by MNL-induced interfacial strengthening. At low frequencies, water-induced siloxane bond-breaking at the MPTMS MNLsilica interface limits the interface fracture energy, and no polymer voiding is observed. At intermediate frequencies, the increased interface strength due to diminishing water attack at the crack tip facilitates load transfer to, and plasticity in, the polymer, yielding fatigue fracture energies exceeding the static-loading fracture energy; polymer voiding is observed. Arrested polymer plasticity (no voiding observed) due to curtailed chain mobility at very high loading frequencies leads to a low interfacial fracture energy despite high interfacial strength

the tuning of the maximum toughening magnitude $\Gamma_{\text {Fatigue-peak }}$ and high-frequency toughening limit $v_{\text {Trailing. The use of multiple }}$ polymers with different rheological properties could result in novel $\Gamma_{\text {Fatigue }^{-}} v$ characteristics with multiple peaks, plateaus, valleys, and combinations thereof. Such frequency-dependent phenomena could pave the way for realizing novel composites that respond to select loading magnitude/frequency stimuli by either controllably degrading ${ }^{46,47}$, or self-healing ${ }^{48}$ through polymer plasticity and crosslinking of healing agents released during microvoid growth in the polymers.

In summary, we have shown that functionalizing polymermetal-ceramic structures with a MNL at the metal-ceramic interface can lead to multifold increases in fracture energy at certain loading frequencies, yielding values higher than that obtained during static loading. Nanolayer-induced interfacial strengthening allows load transfer to, and plasticity in, the polymer layer. While a threshold interfacial strength determines the minimum loading frequency for toughening, the magnitude and loading-frequency range of toughening are primarily dependent on polymer rheology. These facets of heterointerfacial mechanics could be harnessed to design, monitor, and control the stability of composite materials for diverse applications including energy and electronics devices ${ }^{46}$, biomedicine ${ }^{47}$, and smartdegrading and self-healing systems ${ }^{48}$.

\section{Data availability}

The data for the figures that support the findings of this study are available in figshare data repository with the identifier(s) 10.6084/ m9.figshare.7154930 (fracture energy data), 10.6084/m9.figshare.7159529 (microscopy data), 10.6084/m9.figshare.7159541 (XPS data), and 10.6084/m9.figshare.7159553 (DMA data).

Received: 7 March 2018 Accepted: 7 November 2018 Published online: 07 December 2018

\section{References}

1. Loehman, R. \& Tomsia, A. Joining of ceramics. Am. Ceram. Soc. Bull. 67, 375-380 (1988)

2. Lane, M. W., Dauskardt, R. H., Krishna, N. \& Hashim, I. Adhesion and reliability of copper interconnects with $\mathrm{Ta}$ and $\mathrm{TaN}$ barrier layers. J. Mater. Res. 15, 203-211 (2000).

3. Yin, Z., Wei, J. \& Zheng, Q. Interfacial materials for organic solar cells: recent advances and perspectives. Adv. Sci. 3, 1500362 (2016).

4. Ohashi, K. L. \& Dauskardt, R. H. Effects of fatigue loading and PMMA precoating on the adhesion and subcritical debonding of prosthetic-PMMA interfaces. J. Biomed. Mater. Res. 51, 172-183 (2000).

5. Kim, J.-K. \& Mai, Y. High strength, high fracture toughness fibre composites with interface control-a review. Compos. Sci. Technol. 41, 333-378 (1991).

6. Tang, L.-G. \& Kardos, J. L. A review of methods for improving the interfacial adhesion between carbon fiber and polymer matrix. Polym. Compos. 18, 100-113 (1997).

7. Suresh, S., Vasudévan, A. K. \& Bretz, P. E. Mechanisms of slow fatigue crack growth in high strength aluminum alloys: role of microstructure and environment. Metall. Trans. A 15, 369-379 (1984).

8. Yi, K. S., Cox, B. N. \& Dauskardt, R. H. Fatigue crack-growth behavior of materials in viscous fluid environments. J. Mech. Phys. Solids 47, 1843-1871 (1999).

9. Kawaguchi, T. \& Pearson, R. A. The moisture effect on the fatigue crack growth of glass particle and fiber reinforced epoxies with strong and weak bonding conditions: part 1. Macroscopic fatigue crack propagation behavior. Compos. Sci. Technol. 64, 1981-1989 (2004)

10. Snodgrass, J. M., Pantelidis, D., Jenkins, M. L., Bravman, J. C. \& Dauskardt, R. H. Subcritical debonding of polymer/silica interfaces under monotonic and cyclic loading. Acta Mater. 50, 2395-2411 (2002).

11. Cannon, R. M., Dalgleish, B. J., Dauskardt, R. H., Oh, T. S. \& Ritchie, R. O. Cyclic fatigue-crack propagation along ceramic/metal interfaces. Acta Metall. Mater. 39, 2145-2156 (1991).

12. McNaney, J. M., Cannon, R. M. \& Ritchie, R. O. Fracture and fatigue-crack growth along aluminum-alumina interfaces. Acta Mater. 44, 4713-4728 (1996).

13. Yan, Y., Sumigawa, T. \& Kitamura, T. Effect of environment on fatigue strength of $\mathrm{Cu} / \mathrm{Si}$ interface in nanoscale components. Mater. Sci. Eng. A 556, 147-154 (2012).

14. Sharratt, B. M., Wang, L. C. \& Dauskardt, R. H. Anomalous debonding behavior of a polymer/inorganic interface. Acta Mater. 55, 3601-3609 (2007).

15. Paris, P. \& Erdogan, F. A critical analysis of crack propagation laws. J. Basic Eng. 85, 528 (1963).

16. Ramanath, G. et al. Self-assembled subnanolayers as interfacial adhesion enhancers and diffusion barriers for integrated circuits. Appl. Phys. Lett. 83, 383 (2003).

17. Gandhi, D. D. et al. Annealing-induced interfacial toughening using a molecular nanolayer. Nature 447, 299-302 (2007).

18. Jain, A. et al. Atomistic fracture energy partitioning at a metal-ceramic interface using a nanomolecular monolayer. Phys. Rev. B 83, 35412 (2011).

19. Kwan, M., Braccini, M., Jain, A., Lane, M. W. \& Ramanath, G. Interplay between bond breaking and plasticity during fracture at a nanomolecularlymodified metal-ceramic interface. Scr. Mater. 121, 42-44 (2016). 
20. O’Brien, P. J. et al. Bonding-induced thermal conductance enhancement at inorganic heterointerfaces using nanomolecular monolayers. Nat. Mater. 12, 118-122 (2013).

21. Cardinal, T., Devender, F., Borca-Tasciuc, T. \& Ramanath, G. Tailoring electrical transport across metal-thermoelectric interfaces using a nanomolecular monolayer. ACS Appl. Mater. Interfaces 8, 4275-4279 (2016).

22. Cardinal, T., Kwan, M., Borca-Tasciuc, T. \& Ramanath, G. Effect of molecular length on the electrical conductance across metal-alkanedithiol-Bi2Te3 interfaces. Appl. Phys. Lett. 109, 173904 (2016).

23. Cardinal, T., Kwan, M., Borca-Tasciuc, T. \& Ramanath, G. Multifold electrical conductance enhancements at metal-bismuth telluride interfaces modified using an organosilane monolayer. ACS Appl. Mater. Interfaces 9, 2001-2005 (2017).

24. Ramanath, G. et al. Tuning of noble metal work function with organophosphonate nanolayers. Appl. Phys. Lett. 105, 81601 (2014).

25. Kwan, M., Cardinal, T., Mutin, P. H. \& Ramanath, G. Work function tuning at Au-HfO2 interfaces using organophosphonate monolayers. Appl. Phys. Lett. 108, 191607 (2016).

26. Ganesan, P. G., Singh, A. P. \& Ramanath, G. Diffusion barrier properties of carboxyl- and amine-terminated molecular nanolayers. Appl. Phys. Lett. 85, 579 (2004).

27. Gandhi, D. D. et al. Molecular-nanolayer-induced suppression of in-plane $\mathrm{Cu}$ transport at Cu-silica interfaces. Appl. Phys. Lett. 90, 163507 (2007).

28. Gandhi, D. D., Singh, A. P., Lane, M., Eizenberg, M. \& Ramanath, G. Copper diffusion and mechanical toughness at $\mathrm{Cu}$-silica interfaces glued with polyelectrolyte nanolayers. J. Appl. Phys. 101, 84505 (2007).

29. Garg, S. et al. Metal-dielectric interface toughening by molecular nanolayer decomposition. J. Appl. Phys. 108, 34317 (2010).

30. Yu, K. J., Yan, Z., Han, M. \& Rogers, J. A. Inorganic semiconducting materials for flexible and stretchable electronics. npj Flex. Electron 1, 4 (2017).

31. Mir, S. H. et al. Review-organic-inorganic hybrid functional materials: an integrated platform for applied technologies. J. Electrochem. Soc. 165, B3137-B3156 (2018).

32. D’Elia, E., Eslava, S., Miranda, M., Georgiou, T. K. \& Saiz, E. Autonomous selfhealing structural composites with bio-inspired design. Sci. Rep. 6, 25059 (2016).

33. Charalambides, P. G., Lund, J., Evans, A. G. \& McMeeking, R. M. A test specimen for determining the fracture resistance of bimaterial interfaces. $J$. Appl. Mech. 56, 77 (1989).

34. Ma, Q. A four-point bending technique for studying subcritical crack growth in thin films and at interfaces. J. Mater. Res. 12, 840-845 (1997).

35. Ramanath, G. et al. Self-assembled subnanolayers as interfacial adhesion enhancers and diffusion barriers for integrated circuits. Appl. Phys. Lett. 83, 383 (2003)

36. Vlassak, J. J., Lin, Y. \& Tsui, T. Y. Fracture of organosilicate glass thin films: environmental effects. Mater. Sci. Eng. A 391, 159-174 (2005).

37. Zhang, X., Bogorin, D. F. \& Moy, V. T. Molecular basis of the dynamic strength of the sialyl Lewis X-selectin interaction. Chemphyschem 5, 175-182 (2004).

38. Schmidt, S. W., Beyer, M. K. \& Clausen-Schaumann, H. Dynamic strength of the silicon-carbon bond observed over three decades of force-loading rates. $J$. Am. Chem. Soc. 130, 3664-3668 (2008).

39. Schmidt, S. W., Pill, M. F., Kersch, A., Clausen-Schaumann, H. \& Beyer, M. K. Mechanically induced silyl ester cleavage under acidic conditions investigated by AFM-based single-molecule force spectroscopy in the force-ramp mode. Faraday Discuss. 170, 357-367 (2014).

40. Chung, J. Y., Nolte, A. J. \& Stafford, C. M. Surface wrinkling: a versatile platform for measuring thin-film properties. Adv. Mater. 23, 349-368 (2011).

41. Whitesides, G. M., Bowden, N., Brittain, S., Evans, A. G. \& Hutchinson, J. W. Spontaneous formation of ordered structures in thin films of metals supported on an elastomeric polymer. Nature 393, 146-149 (1998).
42. Jiang, W. et al. Loading rate dependence of mode II fracture behavior in interleaved carbon fibre/epoxy composite laminates. Appl. Compos. Mater. 8, 361-369 (2001)

43. Todo, M., Takahashi, K., Béguelin, P. \& Kausch, H. H. Strain-rate dependence of the tensile fracture behaviour of woven-cloth reinforced polyamide composites. Compos. Sci. Technol. 60, 763-771 (2000).

44. Kusaka, T., Horikawa, N. \& Masuda, M. Low-velocity impact fracture behaviour of impact-resistant polymer matrix composite laminates under mixed mode loading. Le. J. Phys. IV 10, Pr9-317-Pr9-322 (2000).

45. Vu-Khanh, T. \& Fisa, B. Impact fracture of glass-flake reinforced polypropylene. Polym. Compos. 7, 375-382 (1986).

46. Hernandez, H. L. et al. Triggered transience of metastable poly (phthalaldehyde) for transient electronics. Adv. Mater. 26, 7637-7642 (2014).

47. Kang, S.-K. et al. Bioresorbable silicon electronic sensors for the brain. Nature 530, 71-76 (2016).

48. Patrick, J. F., Robb, M. J., Sottos, N. R., Moore, J. S. \& White, S. R. Polymers with autonomous life-cycle control. Nature 540, 363-370 (2016).

\section{Acknowledgements}

This work was partially supported by funding support from the National Science Foundation through CMMI 1100933/926 and ECCS 1002282/301 grants. We gratefully acknowledge Aditya Prasad and Steven Lee for help in characterizing the rheological properties of the polymer, Jackson Wong for carrying out some of the experiments, and Professor Linda Schadler for stimulating discussions.

\section{Author contributions}

M.K. and M.B. carried out the experimental work and data analyses. G.R. and M.W.L. conceived the project, designed the experiments, and supervised and contributed to, the execution of the experiments, data analyses, and paper writing.

\section{Additional information}

Supplementary Information accompanies this paper at https://doi.org/10.1038/s41467 018-07614-y.

Competing interests: The authors declare no competing interests.

Reprints and permission information is available online at http://npg.nature.com/ reprintsandpermissions/

Publisher's note: Springer Nature remains neutral with regard to jurisdictional claims in published maps and institutional affiliations.

Open Access This article is licensed under a Creative Commons Attribution 4.0 International License, which permits use, sharing, adaptation, distribution and reproduction in any medium or format, as long as you give appropriate credit to the original author(s) and the source, provide a link to the Creative Commons license, and indicate if changes were made. The images or other third party material in this article are included in the article's Creative Commons license, unless indicated otherwise in a credit line to the material. If material is not included in the article's Creative Commons license and your intended use is not permitted by statutory regulation or exceeds the permitted use, you will need to obtain permission directly from the copyright holder. To view a copy of this license, visit http://creativecommons.org/ licenses/by/4.0/.

(c) The Author(s) 2018 